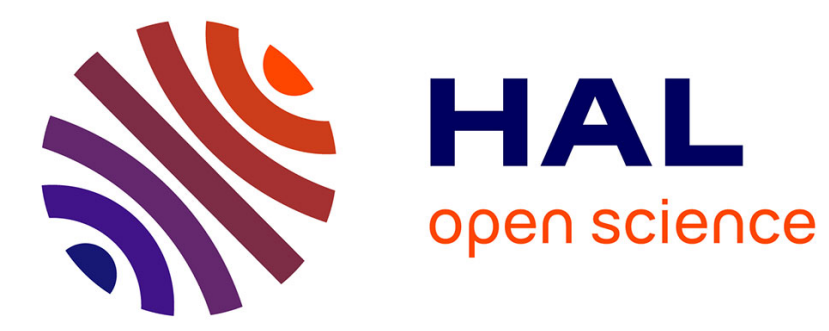

\title{
Experimental determination of the propagation of wireless signals on board a cruise ship
}

Andrea Mariscotti

\section{To cite this version:}

Andrea Mariscotti. Experimental determination of the propagation of wireless signals on board a cruise ship. Measurement, 2011, 44 (4), pp.743-749. 10.1016/j.measurement.2011.01.002 . hal-02959504

\section{HAL Id: hal-02959504 \\ https://hal.science/hal-02959504}

Submitted on 6 Oct 2020

HAL is a multi-disciplinary open access archive for the deposit and dissemination of scientific research documents, whether they are published or not. The documents may come from teaching and research institutions in France or abroad, or from public or private research centers.
L'archive ouverte pluridisciplinaire HAL, est destinée au dépôt et à la diffusion de documents scientifiques de niveau recherche, publiés ou non, émanant des établissements d'enseignement et de recherche français ou étrangers, des laboratoires publics ou privés. 


\title{
Experimental determination of the propagation of wireless signals on board a cruise ship
}

\author{
A. Mariscotti \\ Dipartimento Ingegneria Elettrica, Università di Genova, Via all'Opera Pia, 11A, 16145 Genova, Italy
}

A. Mariscotti, "Experimental determination of the propagation of wireless signals on board a cruise ship," Measurement, Vol. 44, No. 4, May 2011, pp. 743-749. doi: 10.1016/j.measurement.2011.01.002

\begin{abstract}
The characterization of the wireless channel on board a cruise ship is considered by means of frequency domain measurements of received power and propagation attenuation. This electromagnetic environment is rather new and is characterized by abundance of reflecting and diffracting elements, so that a significant difference with respect to more common residential and office environments was found. The measurement setup is simple and easy to position and operate; the measurement results were validated by comparison with another independent measurement setup. The results indicate that the power losses for corridors are not a power law of distance, due to scattering; even in large environments such as restaurant halls the presence of reflecting and scattering objects and installations largely affects the received power.
\end{abstract}

Keywords: Electromagnetic measurements, Indoor propagation channel, Path loss model, UHF measurements, Wireless propagation

\section{Introduction}

There are several services and functions that may require a wireless connection on board ships; besides the interconnection of distributed devices for environmental monitoring, fire alarm and anti-intrusion systems, for passenger ships other services may extend to passenger information systems and support to personal mobile terminals, such as phones, netbooks, laptops, etc. The on board electromagnetic environment was treated in a few works in the past, dedicated to military ships [1]-[4], where some results for the frequency range of wireless transmission (between nearly 800 and $3000 \mathrm{MHz}$ ) are reported and that are considered in the following. The present work differs mainly in having investigated propagation over a far longer distance and for different radiation patterns and polarizations. The most known and studied environments are offices, malls, schools and campus areas, characterized by (partially) absorbing materials, quite large spaces, medium height to high ceilings. For these environments several models have been proposed (deterministic and statistic) and there are databases of parameters and experimental results [5].

A general discussion of deterministic and statistic models for the present application may be found in [6]. 
The models found in the literature often refer to residential and office environments and the most general ones, that may be tuned to different environments, need specific parameters of the same environment, and thus complex measurements. Furthermore, statistical models involving channel response require time domain measurements and processing with high performance equipment. Several wideband disturbances and the presence of other wireless and radio communication devices have prohibited the use of time domain techniques: even using a high energy impulse (produced by a $1 \mathrm{kV}$ peak pulse generator), the captured pulses were disturbed by extraneous waveforms that made difficult or even impossible to measure the time delay and thus the propagation delay of the incoming rays.

A simple and affordable method based on a frequency domain measurement technique is considered, with a quick installation procedure, robust to external disturbance and with results that may be easily interpreted. The reported results for narrow spaces on board deviate from the free space loss law, that says that the power density decreases with the square of distance from the source and it is typical of the far field propagation assumption.

\section{Problem statement}

The target environment is that of a cruise ship and its publicly accessible spaces, in particular halls and corridors. Halls are characterized by one or two decks clearance, large surfaces (more than $1000 \mathrm{~m}^{2}$ ), several scattering objects, such as tables, chairs and columns. Corridors have a variable width between 1 and $1.5 \mathrm{~m}$, depending on the arrangement of cabin doors, passenger ways and service doors, and behave like a large waveguide; the ceiling is not flat and contains edges and ridges, where lighting is also located.

The present analysis is justified since, not only these two environments are representative of the public spaces on board, where wireless services are mostly required, but they are also characterized by extensive use of steel for walls, floor and ceiling, and aluminum for doors, handrails, panels, etc. Moreover, this electromagnetic environment has shown to be peculiar and never investigated before to authors' knowledge.

The analyzed characteristics are in relationship with a straightforward deterministic model of channel path loss for wireless services in the $2.4 \mathrm{GHz}$ frequency band. The attention is on the average signal strength for different distances and positions with respect to the transmitter, on the uniformity of the signal strength around a given measuring point and on the influence of common objects. The definition of this analysis scenario has been done keeping in mind the purpose of wireless facilities for telephone calls, messaging and entertainment.

Two open issues have been addressed by the foregoing analysis, concerning the real behavior of the path loss law (in terms of attenuation versus distance) and the relevance of the multipath terms in terms of signal amplitude and reception at the receiver. The expected path loss law for free space propagation is a power law with exponent two, directly related to the decay of the power density versus the distance from the source. Multipath terms may both degrade signal quality, because of superposition to adjacent symbols and resulting inter-symbol interference, and reduce the measured average power significantly, simply because the local 
power is not uniform across a section orthogonal to the direction of arrival of the main ray from the signal source. This second aspect will be verified with the help of the measurement results in Section 4 .

\section{Measurement system}

The measurements have been performed in the frequency domain using different measuring setups, to evaluate the relevance of multipath terms. The attention was on an easy to operate and easy to install measurement system, with the possibility of verifying the correctness of the results by comparison of independent methods. To this aim three different techniques have been used:

1) a omni-directional antenna driven by an Access Point (AP) locked onto an 802.11 channel (channel 6 at $\left.f_{0}=2437 \mathrm{MHz}\right)$ with transmitter power $P_{t}=100 \mathrm{~mW}(20 \mathrm{dBm})$ and Orthogonal Frequency Division Multiplexing (OFDM) modulation (cycling over the channel bandwidth among a list of clearly distinguishable narrowband sinusoidal components, that may be directly measured with a spectrum analyzer); the receiver is either

1a) a laptop equipped with a wireless card and a diagnostic software (named in the following Laptop Analyzer, LA), or

1b) a Spectrum Analyzer (SA) connected to an omni-directional antenna reading the received power at $f_{0}$ with a max-hold setting and 5 minutes dwell time (the time required to cycle over an OFDM frequency pattern);

2) a directive antenna (log-periodic antenna) fed by a RF generator sourcing a $2.5 \mathrm{GHz} 6 \mathrm{dBm}$ sinusoidal tone; the received signal is read for vertical and horizontal polarizations using the same SA and an omnidirectional antenna; the source has larger directivity to select a smaller amount of signal components directed with an unfavorable angle towards corridor walls and to better understand the channel behavior with respect to multipath components.

Two different omni-directional antennas have been used:

i) a custom sleeve dipole, obtained from a low loss coaxial cable, by cutting a piece of the insulating cover and folding down a length $l$ of the shield, thus leaving uncovered an equal length of the inner conductor; the length is tuned to the frequency $f_{0}$ so that the gain and the input impedance may be computed a priori [7];

ii) a wireless antenna for use with AP, that is a matched whip antenna (a helix antenna in the broadside mode) with a radiation patter and a gain very similar to that of a dipole [8].

For the considered wavelength $\lambda \cong 8 \mathrm{~cm}$, the positioning of the antenna system and the clearance around the antenna itself are two critical aspects, with respect to: first, electromagnetic field uniformity over a vertical section orthogonal to the direction of arrival (the line joining the transmitter and the receiver); second, the multipath fading phenomenon, influenced also by the measuring equipment and operator themselves. For this reason the measurement procedure requires at each measurement location that the SA and the operator are at least $3 \mathrm{~m}$ on the opposite side of the transmitter (this applies to the methods $1 \mathrm{~b}$ and 2). The same thing cannot 
be ensured when the laptop and its internal WiFi antenna are used (method 1a) and in this case the presence of the laptop itself must be accepted and considered.

The main characteristics of the measuring equipment are [7][8]:

- the log-periodic antenna (Schwarzbeck mod. ESLP 9145 [10]) has a gain of $6.79 \mathrm{dBi}$ at $2.5 \mathrm{GHz}$, while the omni-directional antennas have a $2.2 \mathrm{dBi}$ gain [7][8]; the results obtained with the omnidirectional sleeve dipole antenna have been corrected for the input impedance and related reflection coefficient;

- the SA is battery supplied and all the uncertainty terms related to the input reflection coefficient, the input attenuator, linearity and the internal noise are well within the observed variability of the received electromagnetic field.

The uncertainty related to the measuring equipment and to the determination of calibration factors is considered and reported in the following.

Method 1a). The laptop internal wireless antenna is considered equivalent to a dipole for directivity (and gain). The remaining measurement chain inside the laptop itself has no certificate or datasheet, thus it is considered matched and calibrated within an acceptable uncertainty of few $\mathrm{dB}$, confirmed by the readings on another laptop used for reference and placed in the same position at the laboratory before on site measurements (substitution method).

Methods 1b) and 2). The SA is an Agilent mod. N1996A [11], for which the manufacturer datasheet and the calibration certificate state:

- displayed average noise level $-152 \mathrm{dBm} / \mathrm{Hz}$; for the used Resolution Bandwidth (200 kHz) the displayed level was always more than $20 \mathrm{~dB}$ below the measured input signal, with a negligible contribution to overall uncertainty; the use of $200 \mathrm{kHz}$ is a compromise between a short sweep time and an adequate frequency resolution to detect each OFDM component;

- overall amplitude accuracy (95\% confidence) $\pm 0.6 \mathrm{~dB}$ (this is the main accuracy contribution);

- scale fidelity $\pm 0.2 \mathrm{~dB}$ for the measured input levels.

The two small antennas have similar radiation patterns by theory (and thus similar gains) and the different input impedance and thus reflection coefficient has been numerically corrected during post-processing. Taking a dipole as the reference antenna [7], the effective aperture at resonance is given by $A_{e}=0.13 \lambda^{2}=0.002$, with a correction factor $k_{A e}=27 \mathrm{~dB}$. The uncertainty of this correction factor has been estimated by accounting for frequency (with respect to $f_{0}$ ) and antenna geometry deviations, and the result is smaller than $1 \mathrm{~dB}$. The input impedance again at resonance for a length/diameter ratio of 25 is $Z_{i n}=88+\mathrm{j} 27 \Omega$ and the correction factor on the measured power for the reflection coefficient is $k_{\mathrm{VswR}}=3 \mathrm{~dB}$. The length of the used RG400 cable was $2.5 \mathrm{~m}$ and cable losses factor $k_{\text {cable }}$ amounts to less than $0.7 \mathrm{~dB}$.

The directive log-periodic antenna is characterized by antenna factor and gain that are not accompanied by an explicit accuracy statement on the manufacturer datasheet, but it is known that Schwarzbeck has one of the 
lowest calibration uncertainty, lower than $1 \mathrm{~dB}$ with $k=2$ coverage factor. In general the calibration methods are different and it is known that they may differ by a worst case uncertainty of $\pm 4 \mathrm{~dB}$ may be foreseen [9].

Finally, as it will be shown by inspection of the results in the next Section, the data dispersion due to changes of antenna position and the effects of multipath fading is much larger than the equipment uncertainty.

\section{Measurement Results}

The presented results aim at first, validating the measurements obtained with either of the three methods by direct comparison, and, second, deriving a law of propagation for the typical environments of a cruise ship, corridors and large halls.

\subsection{Large space: restaurant hall}

Measurements were taken at a restaurant hall located on two decks, with the AP located on the upper deck, close to the main entrance (see Fig. 1, where the AP label is repeated for reference also on the lower deck on the right).

\section{Figure 1}

The numbers at the $i$-th measurement point indicate the $x_{i}$ and $y_{i}$ distance in the deck horizontal plane with respect to the AP position; the difference in height $z_{i}$ (along the vertical coordinate $z$, not shown) takes into account the distance between decks (approximately $3 \mathrm{~m}$ ), the height above ground of the antenna $(1 \mathrm{~m}$ for the measurements in the restaurant hall) and the height of the AP, secured with adhesive tape to a door profile at $2.10 \mathrm{~m}$ height. The vertical viewing angle $\theta$ used in the following is given by

$$
\theta=\arctan \left(z_{i} / \sqrt{x_{i}^{2}+y_{i}^{2}}\right)
$$

The power density values, recalculated by applying gain, effective aperture and corrective factors to the measured power at the points 1 through 19, are shown in Fig. 2.

\section{Figure 2}

The properties and distribution of the measured point appearing in Fig. 2 may be briefly commented. The values of Fig. $2 b$ correct the different vertical viewing angles of the measurement points with respect to the $\mathrm{AP}$ and the reduction of directivity with respect to the direction of maximum, indicated by the respective radiation patterns.

The points $01,02,03$ and 04 show a decay with distance that is almost corresponding to the expected free space path loss decay; going from point 1 to point 2, the distance is increased by approximately $20 \%$ and the power density reduction is exactly $2 \mathrm{~dB}$ (while the reciprocal of the square of the $20 \%$ increase is $1 / 1.44=0.69$ that is a $31 \%$ reduction, so $1.6 \mathrm{~dB}$ ). Going from point 01 to point 03 the distance is increased by $60 \%$ and the observed power density reduction is almost $9 \mathrm{~dB}$, slightly larger than the expected $4.5 \mathrm{~dB}$ (the distance ratio is 1.68), because of the increased scattering due to some interposed columns and handrails. For points 04,05 and 06 the attenuation is about $6.5 \mathrm{~dB}$ only 1-1.5 dB larger than the expected free space loss (approximately 4.8 for point 04 and 06 and $5.7 \mathrm{~dB}$ for point 05 ). 
Points 04* was left and represents a small positioning error with respect to the twin point 06 (point 04 indicates the correct position): the scattering of a column in front of the measurement point had a relevant effect with a net loss of $4 \mathrm{~dB}$.

Point 08 , immediately below the AP, at a short distance but separated by a deck floor (light type), exhibits a power density larger than the one that could be obtained for a continuous heavy steel deck.

Points 10,11, 12 and 13 have a similar sequence and spatial location as points 01, 02, 03 and 04, but they are located on the lower deck with respect to the AP and because of the presence of the deck floor the received power density is lower; at point 13, the line-of-sight is again ensured and the received power density increases to a value closer to the one measured for similar points 04 and 06.

Points 16, 17, 18 and 19 are points in front of the AP with different situations of obstacles and angles: while 17,18 and 19 almost meet the free space losses law, point 16 is experiencing partial obstruction of a column, with a loss of $7 \mathrm{~dB}$ with respect to point 17 .

Point 07 - between the two decks amid the descending stair - is the best position because of the line-ofsight view of the AP without interfering objects nor reflections from the floor and furniture.

\subsection{Narrow space: cabins corridor}

The analysis of the measurements taken in a cabins corridor revealed a significant multipath reflection and a peculiar path loss law. Measurements were taken at two different heights above ground (1 $\mathrm{m}$ and $1.5 \mathrm{~m})$ and three horizontal positions (centre of the corridor and $\pm 0.4 \mathrm{~m}$ from the centre, with a minimum distance from the walls of $0.2 \mathrm{~m}$ ), as shown in Fig. 3. The points were selected to simulate the positions in space of a handheld terminal or netbook.

\section{Figure 3}

In Fig. 4 all the measurement results are collected together as robust averages curves (obtained by discarding $33 \%$ of the outlying values by means of the trimmean function in Matlab) with dispersion intervals, determined as the sample deviation standard on the set of all measurements at each location. The number of the repeated measurements was variable: four for method $1 \mathrm{~b}$, six for method 1a, two for each method 2 curve.

\section{Figure 4}

From Fig. 4 we see that the data dispersion is larger for omni-directional sourcing (obtained with the AP equipped with its own antenna). At some distances data dispersion is comparable also for the more directive method 2, but this is due to the specific geometry of the measurement location (hand rails, edges, more doors). Since the data were collected as both the six measurement points on the cross section at a given position and repeated measurements at the same point and the same position along the corridor, it was possible to evaluate separately the dispersion $\sigma_{m}$ of the measured received power due to multipath and the repeatability $\sigma_{r}$ of the measurement procedure. The dispersion $\sigma_{m}$ over the six points by discarding the two outliers is always between 3 and $5 \mathrm{~dB}$, while the repeatability $\sigma_{r}$ for four and six repeated measurements is normally between 1 and $2 \mathrm{~dB}$ and only $30 \%$ of times above $2 \mathrm{~dB}$ (the maximum over 26 positions is $3 \mathrm{~dB}$, the average is $1.8 \mathrm{~dB}$ ). 
The curves shown in Fig. 4 are identified for the used measurement methods. The attenuation curve (or path loss) is quite different for the two methods (see also Fig. 6 below), since in the first the multipath influence is much more significant, while the log-periodic antenna is able to convey the emitted power into a smaller solid angle, thus avoiding a large part of multipath interference in the first part of the corridor. Of course, the geometry of the corridor is peculiar and characterized by a $25 \mathrm{~cm}$ enlargement of the corridor width (normally $1 \mathrm{~m}$ ) at the cabin doors and the presence of handrails and several service doors, some of which locked open at the moment of the measurements; moreover the ceiling is not homogeneous and is full of edges and niches for lighting. For this reason some of the 6 measuring points at each longitudinal position may give a much lower (or higher in some cases) reading than the others, since exposed to the influence of operator's body and its position besides location geometry; from this the need for robust statistical analysis and removal of outliers during data post-processing.

The results shown so far were obtained for vertically polarized field; the comparison of the propagation characteristics vertically and horizontally polarized fields is shown in Fig. 5 for Method $2 b$, thus using a directive source.

\section{Figure 5}

The horizontally polarized field exhibits a larger attenuation versus distance, is much more influenced by the several doors and niches on the lateral walls and doesn't reach any situation of "free propagation", like for the vertical polarization at about $60 \mathrm{~m}$.

The power decay versus distance is obtained from the experimental results by linear interpolation in Fig. 6 , in order to estimate a power law coefficient.

\section{Figure 6}

From Fig. 6(a) we see that the S.A. and the L.A. values are in agreement up to about 60-70 m distance, with the L.A. values larger than S.A. values by what we could call "multipath gain": the S.A. method 1b) measures exclusively the central frequency component using max hold and repeated sweeping over the $f_{0}$ frequency interval, thus excluding multipath components, while the received power calculated by method 1a) is based on the acquisition of the complete channel bandwidth and demodulation of all the signal components. The difference between L.A. and S.A. values ranges from few $\mathrm{dB}$ (for some points at shorter distance) up to 6 $\mathrm{dB}$, quite stable for distance values above about 30-40 $\mathrm{m}$.

Above 60-70 m S.A. values (method 1b) decreases more rapidly than L.A. values (method 1a); a possible explanation may be found in the lateral corridor at $65 \mathrm{~m}$ distance, that probably largely modifies the propagation along the main corridor, so that the multipath terms become a significant percentage of the received signal. The difference between L.A. and S.A. values increases slightly with distance from 6 to $8 \mathrm{~dB}$.

From Fig. 6(b) we see that the slope of the path loss is approximately one half of the previous results and that above $70 \mathrm{~m}$ the interpolating line is almost horizontal, to confirm that the direct and reflected rays have found a favorable propagation mechanism, possibly thanks to the more favorable angle of incidence of the rays emitted by the directive source. 
It is possible to exemplify the power density decay vs. distance as:

$$
\begin{array}{ll}
10 \mathrm{~m} \rightarrow 25 \mathrm{~m}: & 10 \mathrm{~dB} \text { of attenuation } \\
25 \mathrm{~m} \rightarrow 50 \mathrm{~m}: & 10 \mathrm{~dB} \text { of attenuation } \\
50 \mathrm{~m} \rightarrow 75 \mathrm{~m}: & 10 \mathrm{~dB} \text { of attenuation }
\end{array}
$$

This behavior doesn't meet any power-law profile. The confirmation was found by adopting a completely different and independent measurement setup: a log-periodic antenna for the $200 \mathrm{MHz}-1 \mathrm{GHz}$ frequency interval, with a gain of $12 \mathrm{~dB}$ at the frequency of $950 \mathrm{MHz}$ (so more directive than the ESLP 9145) and fed by a $10 \mathrm{~W}$ RF power amplifier. The use of the amplifier was necessary, in order to use a calibrated broadband field meter as the receiving station, since this field meter is designed for human exposure evaluation and has a minimum detectable electric field of approximately $0.1 \mathrm{~V} / \mathrm{m}$. The measurement results shown in Fig. 7 confirm the observed power decay law within $2 \mathrm{~dB}$ : the smaller reduction in the first $25 \mathrm{~m}$ ( $8 \mathrm{~dB}$ against 10 $\mathrm{dB}$ ) is due to the larger directivity (i.e. gain) of the used antenna; then at larger distance (about $50 \mathrm{~m}$ ) we obtain again about $20 \mathrm{~dB}$ of reduction with respect to the $10 \mathrm{~m}$ reference distance, as previously found at 2450 MHz.

\section{Figure 7}

For comparison, the results from [1]-[4] are considered. First of all they refer to much shorter distances, from less than $1 \mathrm{~m}$ to maximum $7 \mathrm{~m}$; second, the path loss attenuation is expressed in different ways. In [1] the path loss attenuation ranges from 7 to $25 \mathrm{~dB} /$ decade for large spaces and only $3 \mathrm{~dB} / \mathrm{dec}$ ade for a narrow environment, confirming a strong "guiding effect". In [4] the attenuation ranges from $10 \mathrm{~dB} / \mathrm{decade}$ at 2.5 $\mathrm{GHz}$ to $15 \mathrm{~dB} / \mathrm{dec}$ ade at $5 \mathrm{GHz}$ for a medium size environment; for the measurements performed in a narrow and long passageway the guiding effect is confirmed without giving any quantitative evaluation. It is underlined that in the presented measurement results the guiding effect is compromised by the many edges along the corridor that increase scattering and disuniformity over any longitudinal cross section: the doors are located in niches, $25-30 \mathrm{~cm}$ beyond the profile of the corridor walls; for each door pair there is a column of approximately circular section; service and fire doors have jambs with not a negligible thickness compared to the wavelength.

It is possible to conclude that the directive generation of wireless signals represents a valid method for the wireless coverage of long reflecting and diffracting corridors.

Concerning the extension of the wireless coverage inside cabins, it was experimentally determined a power loss of about $10 \mathrm{~dB}$ from the outside to the inside due to the cabin door, that may be easily taken into account during coverage study with a suitable margin on the calculations.

\section{Conclusions}

A preliminary analysis of the propagation of wireless signals on board cruise ships is presented. The environment is rather peculiar since there are numerous reflecting surfaces and several areas are characterized by a large scattering due to metallic columns, handrails, niches, doors, edges and corners both on walls and 
ceilings. The characterization of the wireless channel has been done in the frequency domain for large and narrow spaces, namely a restaurant hall and a cabin corridor. Large areas may be characterized by an almost free space law of propagation, while a non-power law of propagation has been found for the corridor.

The measurement setup is simple and easy to position and operate; different antennas may be used to select the amount of multipath components for a given environment geometry (corridor length or hall extension). The results consist of corrected path loss laws for halls and corridors and the quantification of scattering influence. Multipath components as well as the path loss versus distance may be kept under control in the final application if a directive antenna is used as the source antenna of the Access Point.

Another outcome of the measurement campaigns and processing of the measurement results is a simplified tool for the planning of wireless network coverage, that is the assignment of the number and position of Access Points with respect to the geometry of the environment. The tool incorporates the power decay curves, the characteristics of the sourcing and receiving hardware and the margins due to measurement uncertainties and second order effects.

\section{References}

[1] E. Balboni, J. Ford, R. Tingley, K. Toomey and J. Vytal, "An empirical study of radio propagation aboard naval vessels", IEEE Conference on Antennas and Propagation for Wireless Communications, 06-08 Nov. 2000, Waltham, MA, USA, pp. 157-160.

[2] L. Mokole, M. Parent, T.T. Street and E. Tomas, "RF propagation on ex-USS Shadwell", IEEE Conference on Antennas and Propagation for Wireless Communications, 06-08 Nov. 2000, Waltham, MA, USA, pp. 153-156.

[3] D.R.J. Estes, T.B. Welch, A.A. Sarkady and H. Whitesel, "Shipboard radio frequency propagation measurements for wireless networks", IEEE Military Communications Conference MILCOM 2001, 28-31 Oct. 2001, Tysons Corner, McLean, VA, USA, vol. 1, pp. 247-251.

[4] P. Nobles and L.R. Scott, "Wideband propagation measurements onboard HMS Bristol", IEEE Military Communications Conference MILCOM 2003, 13-16 Oct. 2003, Boston, MA, USA, vol. 2, pp. 1412-1415.

[5] T. S. Rappaport, Wireless Communications: Principles and Practice, 2nd ed., Prentice Hall, Upper Saddle River, NJ, 2002.

[6] A. Mariscotti, M. Sassi, A. Qualizza and M. Lenardon, "On the propagation of wireless signals on board ships”, IEEE I2MTC 2010, Austin, TX, USA, May 3-6, 2010

[7] C. Balanis, Antenna Theory: Analysis and design, 2nd ed., John Wiley

[8] R.C. Johnson (ed.), Antenna Engineering Handbook, 3rd ed., McGraw-Hill, 1993.

[9] A. Mariscotti, "Measurement Procedures and Uncertainty Evaluation for Electromagnetic Radiated Emissions from Large Power Electrical Machinery", IEEE Trans. on Instrumentation and Measurement, 56 (6) (2007) 2452-2463.

[10] Schwarzbeck, Antenna Datasheet mod. ESLP 9145

[11] Agilent Technologies, N1996A Manual, March 2007 (5989-3678) 

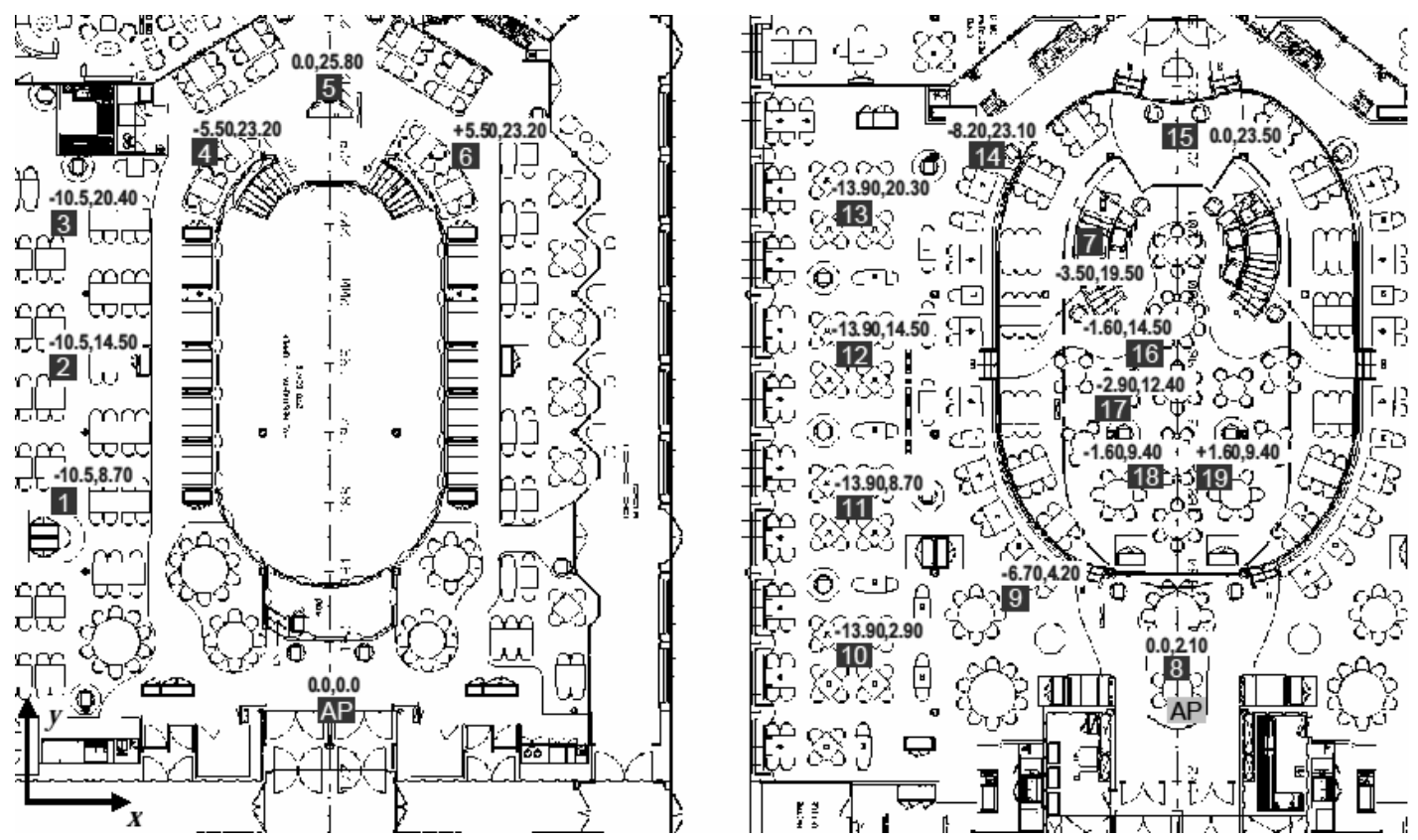

Figure 1. Map of the measurement points (black squares) in the restaurant hall (upper deck \#4, left, and lower deck \#3, right); the $(x, y)$ distance of each point from the AP is shown in metres; the AP was located on deck 4, on deck 3 its footprint is shown for reference 


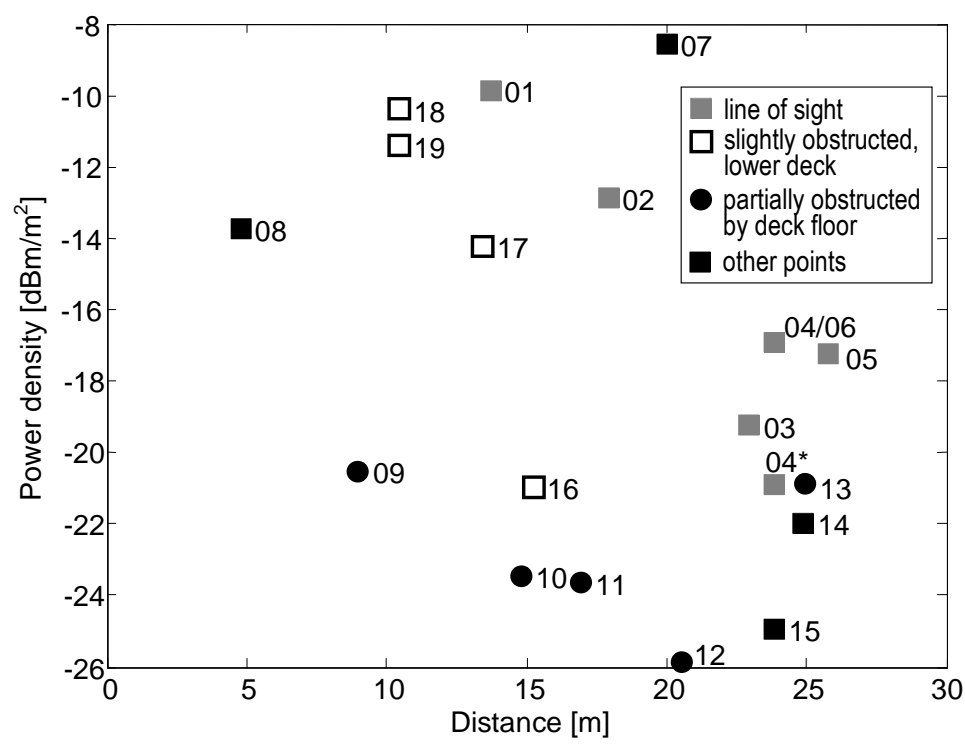

(a)

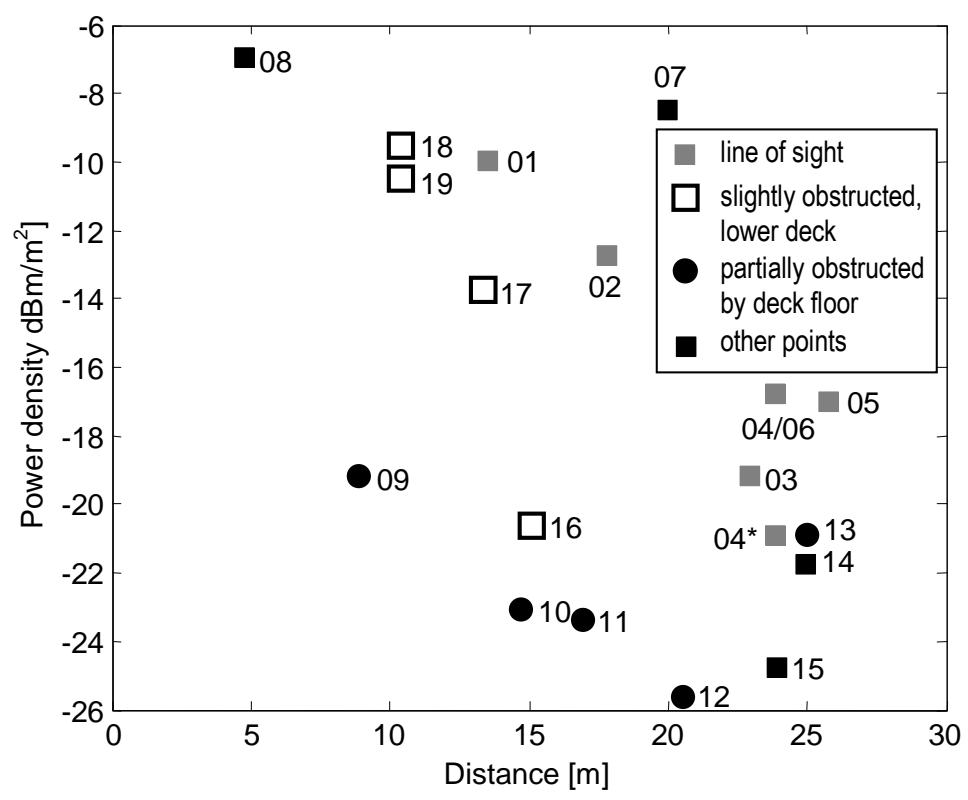

(b)

Figure 2. Power density values vs. distance (method $1 b$ ); the numbers refer to the measuring points of Fig. 1: (a) raw measured data, (b) data corrected for the vertical angle with respect to AP and related radiation patterns. 

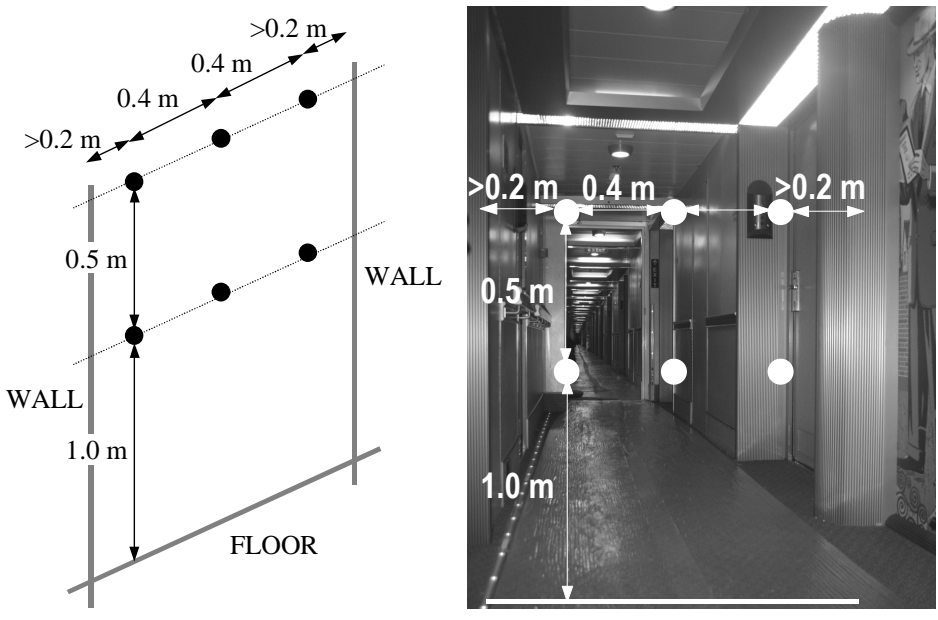

Figure 3. Measurement points at each measurement location

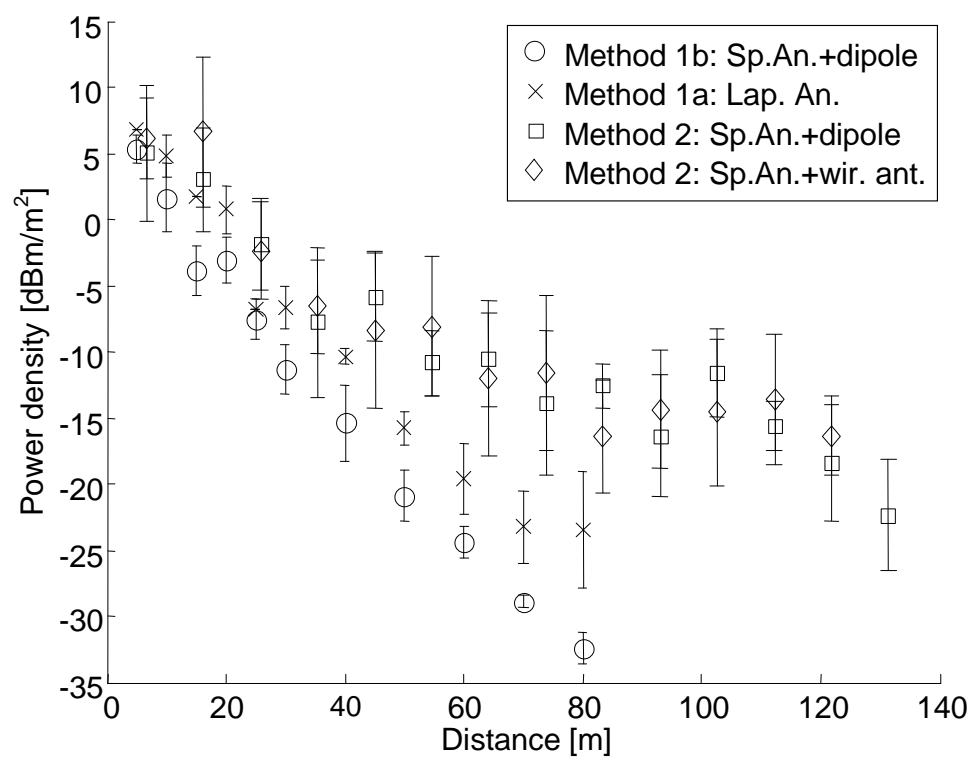

Figure 4. Average power density vs. distance (vertically polarized field) 


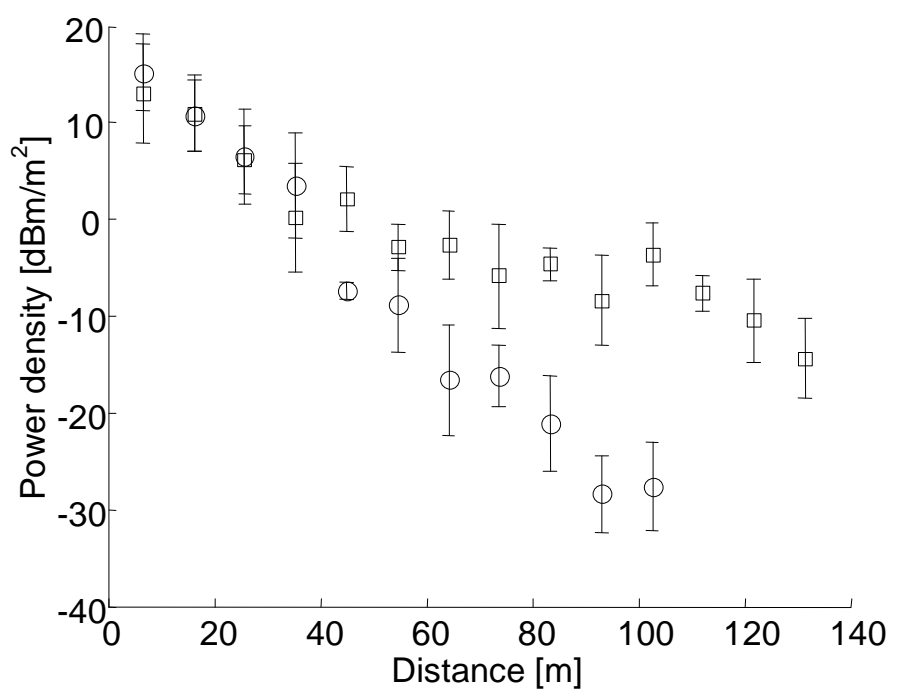

Figure 5. Average power density vs. distance: comparison of vertically (square) and horizontally (circle) polarized field for Method $2 b$

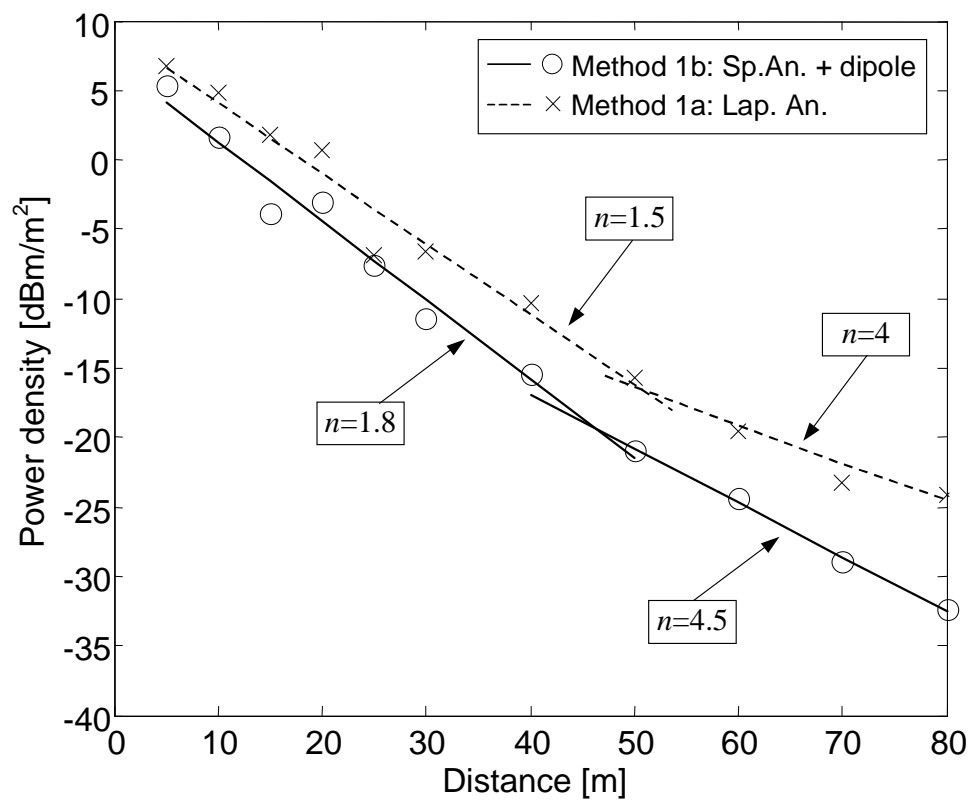

(a) 


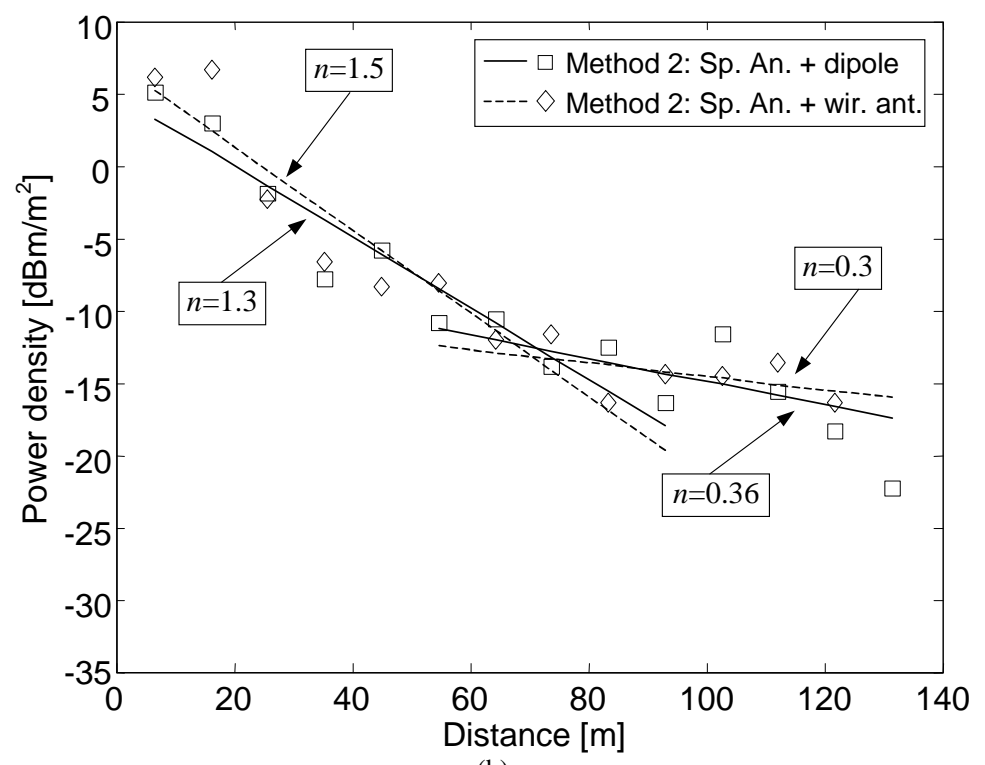

(b)

Figure 6. Average power density values vs. distance and linear interpolation by least mean square regression: (a) omnidirectional sourcing (method 1), (b) directional sourcing (method 2); the approximate exponent $n$ of the power law is shown for each interpolating curve

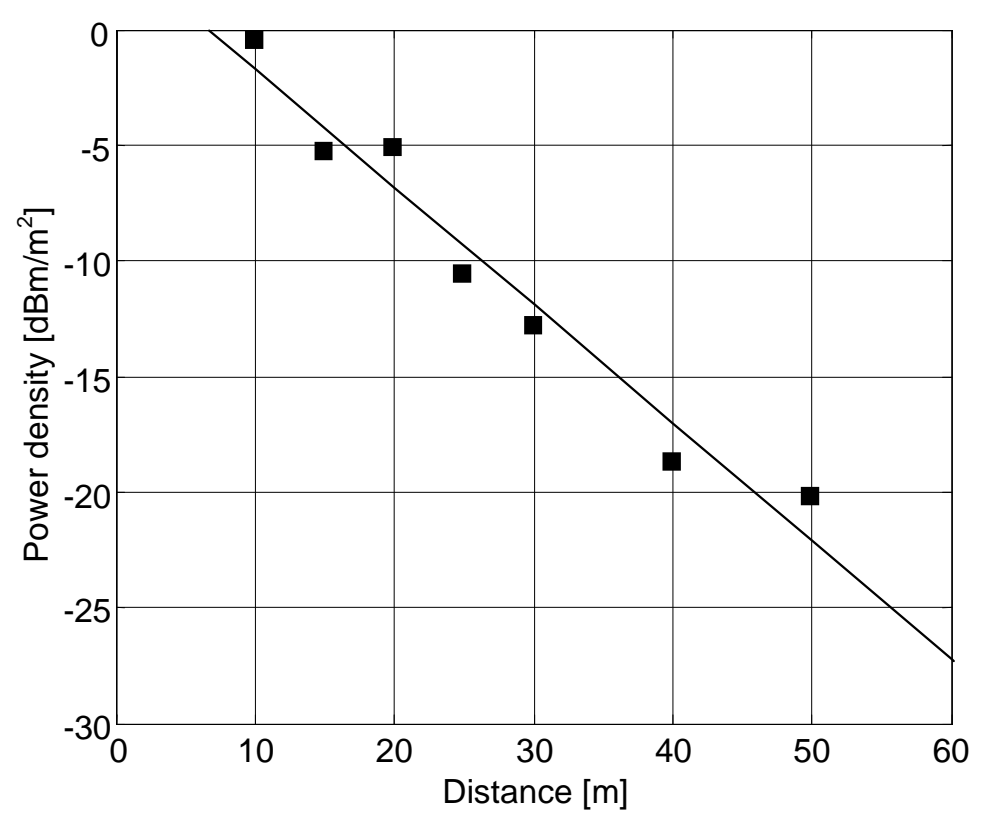

Figure 7. Average received power values vs. distance at $950 \mathrm{MHz}$ (broadband RF field meter) 